\title{
À rebours des systèmes: esthétiques du chaos-monde et de la chaosmose chez Édouard Glissant et Félix Guattari
}

\author{
Hugues Azérad
}

Trouver la juste mesure de mon chaos primordial. En quoi j'échouais sans cesse [...] (oui, je l'engage ici la poésie: qu'elle m'accorde la signification de mon langage, pour témoigner de la signification de mon histoire. ${ }^{1}$

Le chaos n'est pas une pure indifférenciation: il possède une trame ontologique spécifique. Il est habité d'entités virtuelles et de modalité d'altérité qui n'ont rien d'universel [...]. C'est dans le maquis de l'art que se trouvent les noyaux de résistance parmi les plus conséquents au rouleau compresseur de la subjectivité capitalistique, celle de l'unidimensionnalité, de l'équivaloir généralisé, de la ségrégation, de la surdité à l'altérité vraie. $^{2}$

Dès ses tout premiers écrits de la fin des années 1940, Édouard Glissant a lutté contre le système de la colonisation, système alors pleinement validé par la pensée universaliste et républicaine, ${ }^{3}$ en faisant le jour sur les apories et les motivations violentes qui le sous-tendaient. De tous les auteurs des Caraïbes francophones, Glissant est le seul qui soit né

1. Édouard Glissant, Soleil de la conscience (Paris: Gallimard, 1956, réimpr. 1997), p. 48.

2. Félix Guattari, Chaosmose (Paris: Galilée, 1992), pp. 114, 125, désormais $C H$ dans le texte.

3. Sur ce lien toxique, voir Achille Mbembe, Sortir de la grande nuit: essai sur l'Afrique décolonisée (Paris: La Découverte, 2010), pp. 93-171. 
au cœur de ce système, dans une plantation, 'sur l'habitation de Mornedes-Esses, à Sainte-Marie, dans le bruissement des cannes, les obstinations de mulets bâtés, les odeurs de terre et d'herbes, les senteurs de rhum et de sucre', comme le rappellent Chamoiseau et Confiant dans Lettres créoles. ${ }^{4}$ Rappelons aussi que 'le système de la plantation' est l'élément fondateur de la critique glissantienne émise dans son livre théorique princeps, Poétique de la relation. ${ }^{5} \mathrm{~S}$ 'il fallait qualifier l'œuvre de Glissant, on pourrait dire qu'elle est antisystématique de part en part, chaque livre étant une brèche ouverte dans les murs d'une pensée dite occidentale, conquérante, fascinante même, et donc d'autant plus pernicieuse, comme l'indique cette proposition: 'Les pensées de système ou les systèmes de penser furent prodigieusement féconds et prodigieusement conquérants et prodigieusement mortels. ${ }^{6}$ Pour autant, comme 1'a analysé Alexandre Leupin, il ne faut pas prendre cet anti-systématisme opératoire pour un manque de cohérence intrinsèque ou une incapacité à construire un ensemble rigoureux de concepts. Seulement, il faut s'entendre sur ce que concepts et systèmes signifient vraiment chez Glissant:

Glissant est l'un des écrivains les plus systématiques qui soient [...] il y a toujours système, organisation, cohérence, ordonnancement, unité chez lui, fussent-ils ceux d'un chaos [...]. Il faut comprendre la systématicité de l'œuvre [...] comme ouverte, imprévisible et non achevable [...] au contraire d'un système philosophique, qui expulse hors de soi les contradictions que peuvent lui apporter le monde, le système

4. Patrick Chamoiseau et Raphaël Confiant, Lettres créoles: tracées antillaises et continentales de la littérature 1635-1975 (Paris: Gallimard, 1999), pp. 257-58.

5. Édouard Glissant, Poétique de la relation (Paris: Gallimard, 1990), pp. 77-89, désormais $P R$ dans le texte.

6. Édouard Glissant, 'Le Chaos-monde: pour une esthétique de la relation', in Introduction à une poétique du divers (Paris: Gallimard, 1996), pp. 81-107 (p. 17). 
glissantien est ouvert sur les contestations que lui apportent le monde $[\ldots . .]^{7}$

À ce diagnostic, ajoutons que les 'pensées de système et les systèmes de pensée', ${ }^{8}$ pour reprendre cette formulation en chiasme qui scande les écrits glissantiens, représentent pour les écrivains antillais une pensée carcérale de l'Autre. De plus, dans la mouvance des préromantiques anglais (Blake) et allemands (Schlegel, Novalis, Hölderlin), nous savons que toute rébellion contre un système de pensée hégémonique nécessite un contre-système, un instrument de lutte - esthétique et poétique avant tout — capable de déjouer les tactiques adverses qui voudront la faire taire. La création, synonyme autant de pensée de la résistance que de résistance poétique chez Glissant, comme c'était le cas dans les travaux de Deleuze et Guattari à partir des années 1970 et 1980, est justement ce qui lui permettra de ne jamais enfermer son œuvre dans un système absolutisant, qui serait toujours de l'Autre, contre l'Autre, et contre le vivant.

Cet article vise en premier lieu à retracer en détail les composantes anti-systématiques de la pensée glissantienne, qui prend son inspiration dans une nouvelle appréhension de ce que Glissant appelle le chaos-monde, dont il ne cessera de donner des définitions à la fois redondantes et différentes au fil de ses œuvres, en théorie dans ses essais, et en pratique dans ses poèmes, faisant verser chaque genre dans les autres mais privilégiant toujours une nouvelle dialectique de l'oralité et de l'écriture, où chaque terme se verra redéfini selon le principe du tremblement. On aura souvent recours aux notions de parole et d'écriture tremblées, en écho aux séismes, cyclones et raz-demarée qui viennent régulièrement battre l'archipel des Caraïbes, et qui relèvent d'une vision chaotique du monde, mais sans que ce chaos ne soit pur désordre ou simple fascination (ou 'fascines' comme dit

7. Alexandre Leupin, Édouard Glissant, philosophe: Héraclite et Hegel dans le tout-monde (Paris: Hermann, 2016), p. 52. Voir aussi Jean-Pol Madou, Errance et épopée: Glissant, Segalen, Walcott (Caen: Passage(s), 2016), pp. 71-79.

8. Édouard Glissant, La Cohée du Lamentin (Paris: Gallimard, 2005), p. 12. 
Glissant $)^{9}$ pour le catastrophisme ou les sciences du chaos. Pour ce faire, nous puiserons dans la diversité de ses écrits, des essais les plus connus aux poèmes qui s'étendent de 1947 à 1993, mais en faisant fond tout particulièrement sur des écrits moins cités, dont le court texte d'éco-esthétique et d'ethno-poétique que l'on trouve dans un recueil pertinemment intitulé Esthétiques du pire. Cependant, une fois posés les jalons du chaos-monde glissantien, et dans l'esprit de la poétique de la relation, nous ferons intervenir la figure de Félix Guattari et surtout sa théorisation révélée dans Chaosmose, qui nous est apparue comme essentielle pour replacer cette notion de chaos dans son contexte poético-historique, et ce, même si elle a été trop souvent gardée dans l'ombre du grand 'autre', Gilles Deleuze. Ce sont non seulement ces deux univers théoriques et esthétiques, tournant tous deux autour du même axe chaotique, qui seront progressivement mis en relation au long de cet article, mais aussi une certaine politique de l'amitié, secrète celle-là, toute de connivence dans ses alarmes lucides et ses légèretés graves, et dont le modèle ou pattern, pourrait bien ouvrir une nouvelle approche à la fois de chacun de ces penseurs fondamentaux, mais aussi de la beauté tremblée que leur esthétique du chaos semble vouloir annoncer, contre toute attente. ${ }^{10}$

9. Édouard Glissant, Le Discours antillais (Paris: Gallimard, 1981, réimpr. 1997), p. 796. Sur cette géopoétique inspirée du chaos, voir Daniel Maximin, Les Fruits du cyclone: une géopoétique de la Caraïbe (Paris: Seuil, 2006).

10. Voir la parution d'un numéro spécial de Chimères, 90 (2016), entièrement dédié à Édouard Glissant, et qui contient de nouvelles révélations, grâce au témoignage et recherches de Sylvie Glissant en particulier, sur la connivence de pensée et d'écriture entres ces deux penseurs. 
Pensées et esthétiques du chaos-monde

Examinons tout d'abord comment l'appareil glissantien de conceptsnotions (terme plus adéquat que le concept seul, trop hégélien, chaque concept-notion tirant sa validité de son rapport à un autre conceptnotion, sans qu'on puisse en retrancher aucun, et sans qu'aucun ne domine sur un autre), systématique en apparence, se sauve de toute tentation hégémonique par ses principes anti-systématiques. La pensée de l'errance, de la trace, archipélique etc. sont autant de tentatives, toujours inachevées, qui cherchent à dire la multiplicité et la beauté de la Relation et à décrire le chaos-monde. Ce terme de chaos-monde, même si anticipé et "paraphilosophé" ${ }^{11}$ dès Soleil de la conscience, ${ }^{12}$ est développé plus tardivement sous la plume de Glissant, à partir de réflexions menées dans sa Poétique de la Relation et portant sur les liens entre les théories du chaos alors en vogue dans les années 1980 (Thom, Prigogine et Stengers) ${ }^{13}$ et ses propres observations quant aux schémas de connaissance scientifiques, jugés à tort ou à raison trop relativistes, conquérants, et inaptes à saisir les turbulences imprévisibles du monde. À l'opposé de la pensée technologique et économique dominante en Occident, l'idée 'peu à peu se rassembla, qu'on pouvait étudier le Chaos sans succomber au vertige désenchanté de ses incessantes transformations' ( $P R$ 150). Ce n'est évidemment pas la théorie de la relativité que Glissant remet en cause, mais les débordements idéologiques qui l'ont parfois accompagnée, établissant

11. Édouard Glissant, 'Les Poétiques du chaos-monde', in Du Pays au Tout-monde, écritures d'Édouard Glissant, éd. par Elena Pessini (Parma: Universita'degli Studi di Parma, 1995), pp. 139-54 (p. 141), désormais $P A$ dans le texte.

12. Sur ce point, voir l'article de Alessandro Corio, 'Le Lieu tremblant du poème-monde chez Glissant et Heidegger', Karib. Nordic Journal for Caribbean Studies, 1 (2014), 7-53 (pp. 44-50).

13. Il faudrait ajouter à cela les affinités productives entre Glissant et le spiralisme chaotique de Frankétienne: voir Rachel Douglas, Frankétienne and Rewriting: A Work in Progress (Lanham: Lexington Books, 2009) et Glissant 'Le Chaos-monde, l'oral et l'écrit', in Écrire la 'parole de nuit', éd. par Ralph Ludwig (Paris: Gallimard, 1994), pp. 111-29; pour une étude théorique des liens entre littérature et sciences du chaos, voir le numéro 'Écriture et chaos' de Trans-, 6 (2008), http://trans.revues.org/102 [consulté le 17 mars 2017]. 
un pont erroné entre Relativité et relativisme culturel. À l'inverse de l'humanisme bon ton colporté par le relativisme culturel, sa propension à essentialiser l'Autre et à réintroduire des hiérarchisations entre les cultures, la théorie du chaos ressortit à un esprit 'd'enquête' et non de conquête, et se rapproche davantage d'une 'méditation expérimentale des processus de relation' $(P R$ 151). C'est là le lien fécond que perçoit Glissant entre la théorie du chaos et sa propre réflexion poétique sur la 'trame chaotique de la relation' ( $P R$ 158). Pour Glissant, il y a une affinité entre les intuitions de cette théorie et les siennes quant aux turbulences du monde, que ce soit dans ses désastres écologiques, politiques et économiques, ou ses beautés qui ne cessent de surgir au hasard des créolisations, de l'effet de diffraction induit par la multiplicité et l'imprévisible sur l'Un et le déterminé. Le chaos-monde est donc autant le résultat d'une observation directe et politique des désordres du monde aux prises avec les tyrannies de 'l'empire' qui battent alors son plein après 1945, que d'une méditation poétique non sur l'état (qui présupposerait l'être) du monde mais sur l'étant (qui relève de la relation) du monde.

Le chaos-monde correspond analogiquement et sert de justification à la lutte glissantienne contre la pensée de système, lutte qui détourne ce mode de pensée plus qu'il ne le rejette complètement, car une autre manière de nommer/fonder un système qui n'en est pas un, ce serait de le dire chaotique, système 'déterministe erratique et aux variances multiples' comme le dira plus tard Glissant dans un texte intitulé 'Les Poétiques du chaos-monde' ( $P A$ 139). Mode opératoire plutôt que validation externe, la science du chaos sert de métaphore scientifique pour étendre la poétique de la relation à des domaines plus concrets mais aussi plus abstraits: 'le chaos-monde est un des modes du Chaos, objet de cette science' ( $P R$ 151). Glissant se dessaisira bien vite des aspects trop programmables de la théorie du chaos, mais il en garde les hypothèses les plus porteuses pour ses propres intuitions qui ne forment jamais de théorie qui ne soit également poétique ou esthétique. D'où l'insistance sur le besoin d'une forme qui fasse langage, pour éviter l'envers absolu du système que serait le désordre totalitaire, autre forme de néantisation de la vie. Glissant s'entend 
préserver une part d'imaginaire à la poétique de la relation, qui n'est ni pur désordre, ni pure science ou théorie, et une part de dicible dans l'imprédictible du chaos-monde:

Le chaos-monde n'est ni fusion ni confusion: il ne reconnaît pas l'amalgame uniformisé - l'intégration vorace - ni le néant brouillon. Le chaos n'est pas 'chaotique' [...]. Mais son ordre caché ne suppose pas des hiérarchies, des précellences — des langues élues et peuples princes. L'esthétique du chaos-monde [...] globalise en nous et pour nous les éléments et formes d'expression de cette totalité, elle en est l'action et la fluidité, le reflet et l'agent en mouvement. La Relation est ce qui en même temps le réalise et l'exprime. Elle est le chaos-monde qui (se) relate. $(P R$ 108)

Glissant ne réitère pas là ce que la théorie post-moderne avait avancé dans les années 1980 et 1990; au contraire, il prend soin de s'en éloigner, pour la simple raison que le post-moderne 'trouve son motif dans une résurrection formaliste des œuvres et ornements du passé occidental, accommodés à l'actuel magma' ( $P R$ 235). L'ordre et la forme dont parle Glissant ne sont ni créés a priori ni nés d'un système préexistant; ils ne sont ni classiques ni post-modernes, mais ils se rapprochent de la parole baroque qui est la résultante (jamais prédictible) de la poétique de la relation, qui elle-même participe de l'esthétique du chaos: 'La poétique de la Relation (qui est donc une part de l'esthétique du chaos-monde) pressent, suppose, inaugure, rassemble, éparpille, continue et transforme la pensée de ces éléments, de ces formes, de ce mouvement' ( $P R$ 109). La pensée ou esthétique du chaos s'appose aux autres formes de pensées anti-systématiques mentionnées plus haut, et comme elles, elle embrasse l'incertain et l'imprévisible.

Bien entendu, trente ans après ces formulations, elles-mêmes mises plus ou moins à l'écart sans jamais être rejetées, le chaos-monde étant de plus en plus remplacé par la notion de Tout-monde (totalité des peuples et paysages, de toutes les 'régions du monde', totalité sans doute moins comminatoire que le chaos-monde), ces formules peuvent 
frapper par leur naïveté, d'ailleurs pleinement assumée par Glissant, qui s'en prévaut. ${ }^{14} \mathrm{Ce}$ serait pourtant oublier la pénétrante portée politique de cette nouvelle esthétique ou ce mode de connaissance poétique aux apparences si désordonnées et surtout incontrôlables. Le chaos-monde est déjà synonyme d'une 'conscience rebelle' ( $P R \quad 153)$ qui refuse de se plier aux ordonnancements et subordinations (néo)coloniaux tels qu'ils sévissaient exactement dans la seconde moitié du vingtième siècle. ${ }^{15}$ La pensée du chaos vient fracturer les systèmes ordonnés de l'Occident, en révélant leur composition fractale et en ouvrant ainsi la voie à une nouvelle approche esthétique (mais aussi politique et éthique) du monde. Nulle irresponsabilité ici, mais volonté créatrice d'envisager les problèmes selon une autre logique qui ne serait plus adventice mais inhérente aux tremblements et turbulences du monde et des peuples. La poétique de la relation, qui est l'expression du chaos-monde ou sa forme dicible, participe paradoxalement d'une observation méticuleuse des situations réelles et d'un antiréalisme lucide. Ce dernier ne cherche pas à fonder l'illusion d'un 'effet de réel' (rejet poli/poétique de la mimésis passive chez Glissant), ${ }^{16}$ mais à exprimer au plus près les apories identitaires qui existent partout où régissent les croyances en la précellence de l'être, de l'identité racine et de la légitimité d'une communauté à l'exclusion des autres. La poétique de la relation est un souci, une éthique serrée du réel chaotique du monde et non de l'être, du soi foucauldien (sans que l'une n'exclue l'autre cependant si l'on y regarde de plus près).

Glissant remonte aux sources chaotiques du réel, dont le gouffrematrice a été la preuve irréfutable dans l'histoire occidentale: sous l'ordre tyrannique de la plantation régnait de fait un chaos-monde de cultures qui attendait d'être nommé et énoncé — mais qui se vivait ou

14. 'Et je dis ingénus et naïfs au plein sens du terme.' Édouard Glissant avec Alexandre Leupin, Entretiens de Baton Rouge (Paris: Gallimard, 2008), pp. 146-47.

15. Sur cette question d'engagement esthético-politique, voir les analyses de René Ménil dans Antilles déjà jadis (Paris: Jean-Michel Place, 1999), pp. 217-32 et de Celia Britton dans Language and Literary Form in French Caribbean Writing (Liverpool: Liverpool University Press, 2014).

16. Glissant, Discours, p. 774. 
se cherchait, s'inventait ainsi - et qui correspondrait aux dynamiques chaotiques des mouvements culturels forcés ou voulus qui ne cessent d'agir de façon sans cesse croissante. De la tragique 'scission créatrice', ${ }^{17}$ viennent sourdre des dynamiques chaotiques d'exister, de faire et de penser qui sont aussi de nouvelles formes du vivant, totalement inattendues et jamais complètement théorisables: leur nature chaotique prévient tout discours homogénéisant. C'est pour cela que la poétique de la relation est totalisante sans être totalitaire: sa nature poétique, son expression sous forme de poème (qui peut prendre à son tour toutes les formes et tous les genres) la préserve de toute hégémonie, car nul poème n'asservit jamais le langage ou la connaissance. Le poème est toujours le multiple vivant sous l'apparence de l'un et du vrai. La poétique énonce la Relation, et celleci exerce en poétique, ce qui ne l'empêche pas d'être agissante, effective, dans le réel. Ce branchement établi entre la pensée du chaos et la poétique de la Relation est donc crucial pour que cette dernière n'instaure pas un ordre factice sur la totalité du réel et échappe au soupçon de subjectivisme et d'évasion-refuge.

La poétique de la Relation est une manière non systématique de catalyser et mesurer heuristiquement les énergies et rencontres chaotiques du monde, retrouvant dans le chaos un miroir de sa propre dynamique. Ainsi la poétique de la relation est-elle chaotique dans ses résultantes comme dans ses modes de fonctionnement, qui se perçoivent toujours dans l'étendue et l'a-synchronie: si ce chaos fait horreur aux pensées de système, c'est qu'il injecte de l'imprévisible dans le déterminé, de la discontinuité dans le continu, et garde une réserve d'arbitraire qui prémunit contre la raison instrumentale et universaliste, sans pour autant céder à l'irrationalisme ou au relativisme. La poétique, comme la théorie du chaos, reste l'un des modes privilégiés d'investigation du réel chez Glissant, permettant à la fois l'agir, la pensée, et des cristallisations en communauté du 'nous'. Relation et chaos sont synonymes de nouveauté, ${ }^{18} \mathrm{~d}$ 'inouï et d' 'insu',

17. Mbembe, Sortir, p. 84.

18. 'Nous devons cahoter tous les genres [...] je crois que nous inventerons des genres nouveaux.' Édouard Glissant, L'Imaginaire des langues (Paris: Gallimard, 2010), p. 30. 
mais ils contiennent aussi une promesse de communauté et de nouveaux chemins de connaissance:

Dans l'étendue, les formes du chaos-monde (le brassage incommensurable des cultures) sont imprévisibles et devinables. Nous n'avons pas encore commencé de calculer leurs résultantes [...] tant d'inventions éclatées soudain, nées de chocs $[\ldots]$ qui sont la matière fluide, turbulente et obstinée et peut-être ordonnée, de notre commun devenir. (PR 152)

Du chaos-monde à la chaosmose, et vice versa

Cette réalisation que les pensées de système classiques ne pouvaient plus permettre d'appréhender le réel dans toute sa complexité, et que l'imaginaire des arts était à même d'injecter des 'conditions initiales' (PA 146) nouvelles dans les vieux systèmes, apporte une certaine validité aux concepts-notions d'errance et d'erratique que Glissant avait commencé à penser à l'orée des années 1950. Comme il le dit, il n'en 'fait pas [son] rosaire, ni [son] lieu de croyance' (PA 144), mais l'analogie entre sa poétique et la science du chaos cristallise un moment de pensée, lui apporte un soutien dans un contexte où la pensée régnante avalisait des systèmes d'une cohérence implacable. Il y avait pourtant exception, et c'est à Paris qu'une rencontre se fera entre Glissant et Guattari — tout d'abord par le biais de la lecture de Rhizome (1976) puis de Mille Plateaux (1980) —, rencontre qui est loin d'être à sens unique d'ailleurs. Si le lien explicitement fait par Glissant dans Poétique de la Relation, entre son concept-notion d'identité-rhizome et le concept de rhizome de Deleuze et Guattari, est connu et amplement commenté, ${ }^{19}$ le lien avec la pensée du chaos de ces derniers l'est

19. Voir Nick Nesbitt, 'The Postcolonial Event: Deleuze, Glissant and the Problem of the Political', in Deleuze and the Postcolonial, éd. par Simone Bignall et Paul Patton (Edinburgh: Edinburgh University Press, 2010), pp. 103-18 et Lorna Burns, 
beaucoup moins, et c'est lui qui nous mènera au vrai dialogue qui s'établira entre Glissant et la part souvent occultée de la paire DeleuzeGuattari, c'est-à-dire Félix Guattari lui-même, et ses écrits post-Mille Plateaux surtout. ${ }^{20}$ François Dosse, dans sa biographie croisée de Deleuze et Guattari, nous apporte des renseignements précieux à cet égard sur cette rencontre: après une première rencontre sans doute ratée dans les années 1980 ('appelé par Guattari, Jean-Jacques Lebel débarque avec Allen Ginsberg, Édouard Glissant, Paolo Fabbri et Christian Bourgois' $)^{21}$ en raison de la dépression de Guattari, Dosse nous dit que Glissant rencontre à nouveau Guattari à Paris et que là, quelque chose se passe. Glissant, 'sidéré par son intelligence, devient aussitôt son ami: "Je me disais: 'J'entends Socrate. J'entendais la même sagesse, la même ironie, la même âpreté d'approche et bienveillance fondamentale' [...] [Deleuze et Guattari] pensent de manière fractale. C'est une pensée fractale, nomade, errante", ${ }^{22}$ Dosse décrit une influence plutôt unilatérale, alors que ces trois penseurs travaillent simultanément sur des idées affines, en partie liées à la théorie du chaos alors en plein essor, tout en partageant le même souci pour les langues dites 'mineures', les paysages, l'anti-universalisme, l'événement, la différence dans la répétition, une pensée nomade qui soit proche du monde et des peuples opprimés, de l'archipel (et non du

Contemporary Caribbean Writing and Deleuze: Literature Between Postcolonialism and Post-Continental Philosophy (London: Continuum, 2012).

20. Rappelons cependant que dans Qu'est-ce que la philosophie? (Paris: Minuit, 1991), Deleuze et Guattari, fortement influencés par les essais de D.H. Lawrence, ébauchent une esthétique du chaos qui demeure ambivalente: 'l'art n'est pas plus fait de chaos que d'opinion; mais, s'il se bat contre le chaos, c'est pour lui emprunter les armes qu'il retourne contre l'opinion [...] l'art n'est pas le chaos, mais une composition du chaos qui donne la vision ou sensation, si bien qu'il constitue un chaosmos' (pp. 204-05). S'il est vrai que le concept de 'variété chaoïde' (p. 205), synonyme de création esthétique, a partie liée avec l'esthétique du chaos glissantienne, le pas vers le chaos-monde n'est pas encore franchi chez Deleuze-Guattari. Guattari et Glissant franchiront ce pas.

21. François Dosse, Gilles Deleuze Félix Guattari. Biographie croisée (Paris: La Découverte, 2007), p. 501. Remarquons le rôle de passeur/ passant intellectuel et artistique joué par Jean-Jacques Lebel, à qui Glissant dédie une section ('L'Cil dérobé') de Les Grands Chaos, in Poèmes complets (Paris: Gallimard, 1994), pp. 427-40 (p. 428).

22. Dosse, Deleuze, p. 515. 
territoire), ce que Glissant appelle 'l'Autre de la pensée'. Dans La Cohée du Lamentin, Glissant fait un clin d'œil aux deux penseurs français avec lesquels il se sentait le plus complice: 'C'est là un choix poétique de Deleuze et de Guattari, et peut-être en ce qui me concerne je m'arrêterais à penser Mille Jungles ou Mille Cyclones, mais le fondement est le même: là où les géographies des idées, des désirs, des créativités, échappent au Territoire, aux systèmes continentaux, et entrent dans les Archipels.'23

Dans sa comparaison, Dosse s'appuie sur un entretien avec Kristina Kullberg, qui note cette fascination partagée pour la pensée du chaos: 'Le chaos-monde chez Glissant lui sert à désigner la mondialisation qu'il qualifie de mondialité, et sa notion d'opacité est très proche de la notion de singularité chez Deleuze et Guattari.' ${ }^{24}$ Dosse et Kullberg subsument ici la pensée glissantienne sous celle de Deleuze et Guattari, évacuant à la fois la spécificité de Guattari et de Glissant. Glissant avait pourtant déjà révélé son affinité plus profonde avec Guattari dans La Cohée du Lamentin, où il distingue Deleuze, 'penseur de la mondialité' de Guattari, qui 'scrute la mondialisation, il palpe le rugueux des étals standardisés, il devine sous le plus neutre et le plus banal le plus oppressif, il parcourt réellement les mondes, leurs hystéries trop cachées et leurs schizophrénies trop visibles. Il prévoit le pire, d'où il tire sérénité'. ${ }^{25}$

Le penseur du Capitalisme Mondial Intégré, écosophe soucieux d'inventer de nouveaux foyers d'énonciation collectifs et de nouveaux modes de subjectivation, rejoint les conclusions de Glissant dans son Discours antillais et sa Poétique de la Relation, qui sont avant tout des diagnostics d'une situation matérielle et mentale aporétique et vouée à la catastrophe, renforcée par une colonisation invisible qui devient universelle (logique de la consommation effrénée, passivité des sujets, troubles mentaux exacerbés). Étant tous les deux plutôt proches des

23. Glissant, La Cohée du Lamentin, p. 137.

24. Dosse, Deleuze, p. 516.

25. Glissant, La Cohée du Lamentin, p. 139. 
mêmes penseurs (Sartre surtout, mais aussi Fanon), ${ }^{26}$ Glissant et Guattari observent des phénomènes similaires chez leurs 'patients' (nous pensons à l'analyse des déséquilibres, traumas et délires, mais aussi bricolage, inventivité, détournements, dans le Discours antillais en particulier), ${ }^{27}$ et ils s'intéressent à la transversalité des investissements libidinaux au travers des champs aussi distants que la sociologie, le végétal, l'animal etc. 'Chez Guattari', nous dit encore Glissant dans un entretien de 2007, 'il y une profusion charnelle dans le contact, avec les pays et avec les gens [...] une espèce d'excitation de l'imprévisible [...]. Deleuze est plutôt un écrivant et Guattari plutôt un parlant' ${ }^{28} \mathrm{Se}$ sentant proche du Brésil, de l'Afrique, du Japon et du Maghreb, Guattari est le penseur qui aimait passionnément les Radios Libres, vecteurs d'une parole libérée justement, et l'un des premiers intellectuels à critiquer lucidement la montée des racismes et sectarismes identitaires religieux au début des années 1980 en France et dans le monde, en même temps qu'il théorisait une triple écologie à la fois environnementale, sociale et mentale. ${ }^{29}$ Mais il faudra les récentes révélations d'Aliocha Wald Lasowski dans son étude exhaustive sur le milieu intellectuel où évoluait Glissant, et de Sylvie Glissant dans un entretien captivant de la revue Chimères, pour faire

26. Voir Frantz Fanon: écrits sur l'aliénation et la liberté (Paris: La Découverte, 2015), éd. par Jean Khalfa et Robert Young.

27. Sur l'étude des névroses des Martiniquais dans Le Discours antillais, voir les analyses de Leupin, dans Glissant philosophe, pp. 272-75.

28. François Noudelmann, 'Deleuze et Guattari, et vice versa: entretien avec Édouard Glissant', Les Vendredis de la philosophie, France-Culture, 16 novembre 2007. Cité dans Aliocha Wald Lasowski, Édouard Glissant, penseur des archipels (Paris: Pocket, 2015), p. 187. Sylvie Glissant, dans un entretien intitulé 'Conversation autour d'un rêve de Glissant et Guattari', avec Anne Querrien, Lucia Sagradini et Monique Zerbib, soulève brillamment le voile sur l'amitié profonde entre Glissant et Guattari, et sur leurs projets politiques/poétiques communs: 'Je pense [...] que Félix et Édouard voulaient entrer ensemble dans ce dire poétique commun en dépit des différences. Ils peuvent paraître complètement différents, hétérogènes, car ils arrivent de champs différents, mais il y avait entre eux, selon moi, ce désir d'un dire partagé. Je crois que cette rencontre devenait une nécessité qui allait produire quelque chose [...]. Peut-être n'ont-ils même pas eu besoin d'écrire ce livre parce que tout était déjà là.' Chimères, 90 (2016), 19-31 (pp. 28-29).

29. Voir en particulier Félix Guattari, Les Trois Écologies (Paris: Galilée, 1989), pp. 32-35. 
vraiment le jour sur la complicité fertile entre ces deux penseurs. Guattari et Glissant pensent de nouveaux modes de subjectivation dans une transversalité ou Relation mondiale: tous deux s'en prennent au culte universalisant du signifiant chez les (post)structuralistes afin de dégager des ruptures expressives a-signifiantes, des agencements processuels et des vecteurs dissidents de singularisation qui fassent rupture avec les faillites des systèmes pseudo-scientifiques, de la 'subjectivité capitalistique' et de la politique. ${ }^{30}$ Wald Lasowski suggère même qu'un projet commun de livre entre les deux penseurs de la chaosphère devait se faire:

Plus que Gilles Deleuze, Glissant fréquente surtout, on le sait, et pendant longtemps, Félix Guattari. Il discute souvent avec ce penseur-psychiatre, engagé dans le renouvellement de l'approche psychiatrique du sujet. Glissant invite Guattari au centre d'études françaises à Baton Rouge [...]. Les deux amis [...] projettent d'écrire ensemble un livre sur les formes nouvelles de pensée et d'écriture: par rapport à l'oral, qu'est-ce que l'écriture comme spécificité, dans un monde qui épouse le glissement des 'fonds' et des 'plateaux'? [...] on rêve de ce qu'aurait pu être ce livre écrit par le penseur archipélique des créolisations et le penseur schizophrène analytique des mégapoles. ${ }^{31}$

C'est sur la base de ces premières indications que nous souhaiterions maintenant explorer plus avant la connivence entre ces deux penseurs, nous focalisant en particulier sur leur tentative de fonder une esthétique du chaos, ou plus précisément, de la chaosmose, terme qu'ils empruntent tous deux à Joyce, ${ }^{32}$ pour mieux cerner leur rupture quant aux systèmes de pensée, et montrer que leur esthétique du chaos, même

30. Guattari, Les Trois Écologies, pp. 36-37, 44. Sur la critique du 'défilé des signifiants' chez Glissant, voir Leupin, Glissant philosophe, pp. 303-11. Voir aussi les références à Barthes dans Poétique de la Relation, pp. 38, 74 et 181.

31. Lasowski, Glissant, p. 188; sur ce 'livre', voir Sylvie Glissant (n. 28). Rappelons que Glissant dédie son roman Tout-monde (Paris: Gallimard, 1993) à Guattari, disparu en 1992.

32. James Joyce, Finnegans Wake (London: Faber, 1939), 118.21. 
si différente dans leurs modes de fonctionnement interne, est une forme nouvelle d'engagement esthético-politique.

En effet, chez ces deux penseurs, l'esthétique n'a lieu d'être que si elle s'attaque politiquement aux causes, et que si elle influe sur les pratiques en proposant de nouvelles formes de subjectivations, loin de tout humanisme tautologique et bien pensant mais aussi loin des pratiques militantes traditionnelles. La justesse du diagnostic vaut comme analyse politique, puisqu'elle permet de repérer les failles dans les systèmes prégnants, et d'y injecter son virus porteur de projets concrets à tenter non pas de copier mais de 'penser avec', d'accompagner d'autres engagements concrets, d'autres paroles à naître, d'autres mentalités et sensibilités à faire surgir. Guattari indique à multiples reprises cette nouvelle forme d'engagement et de questionnement:

Alors, lancinante, la question revient: comment modifier les mentalités, comment réinventer des pratiques sociales qui redonneraient à l'humanité — si elle l'a jamais eu — le sens des responsabilités, non seulement à l'égard de sa propre survie, mais également de l'avenir de toute vie sur cette planète, celle des espèces animales et végétales, comme celle des espèces incorporelles, telles que [...] les arts, [...] le rapport au temps, l'amour et la compassion pour autrui, le sentiment de fusion au sein du cosmos? (CH 166)

Il faut rappeler que c'est dans Chaosmose, titre du dernier livre paru de Guattari avant sa mort, que celui-ci avait pleinement élaboré l'idée d'un nouveau paradigme éthico-esthétique pour parer aux emmurements identitaires qui règnent aujourd'hui, et qu'il avait devinés avec tant d'acuité dès les années 1980:

[L]a chaosmose [...] rebondit et bourgeonne sur les états de choses, les corps, les foyers autopoiétiques qu'elle utilise à titre de support de déterritorialisation, elle est chaotisation relative à travers la confrontation d'états hétérogènes de la complexité. On a affaire ici à un infini d'entités virtuelles infiniment riche de 
possibles, infiniment enrichissable à partir de processus créateurs. C'est une tension pour saisir la potentialité créative à la racine de la finitude sensible [...] qui fonde le nouveau paradigme esthétique. ( $\mathrm{CH} 156)$

Ce nouveau paradigme esthétique engage un rapport nouveau à la beauté et au chaos. Publiés quasiment au même moment, Poétique de la relation et Chaosmose font état d'une même volonté d'en finir avec les vieux systèmes de pensée (fondés en partie sur des binarités inamovibles telles que la linguistique saussurienne et le structuralisme qui s'en inspire les avaient établies) et de saisir, voire (s')inspirer des beautés qui affleurent parfois des chocs chaotiques entre les cultures. Des affinités profondes se détachent entre le nouveau paradigme esthétique guattarien, qui privilégie les notions de chaos, flux, création, finitude, relation, fonction poétique, anti-universalisme, singularisation dans le respect des différences et de l'hétérogenèse, et l'esthétique glissantienne, qui vise à lever un certain rythme jaillissant des paysages, œuvres et cultures qu'il s'agit de suivre au plus près sans jamais tenter d'en éclairer les enchevêtrements. Allant à l'encontre des idéologies régnantes qui fustigent ou entérinent l'antinomie entre l'art et la praxis, l'art et le non-art, ces deux penseurs considèrent l'esthétique comme un foyer autonome - une autonomie toute moderniste d'inspiration (Joyce, Faulkner, Kafka, Beckett) — de création et de proliférations rhizomiques, l'œuvre d'art étant un agent bio-esthétique qui donne naissance à de nouvelles modalités de la subjectivité, selon une vision transversale qui figure des traces sur les blocs monolithiques des discours dominants.

Pour ce qui nous concerne ici, avançons d'emblée que la mise en relation de ces deux pensées, qu'il faut nommer pensées esthétiques et qui jusqu'à présent ont été masquées respectivement par la présence plus explicite et absorbante de Deleuze, permet de dégager la rigueur et vigueur que donnent ces penseurs à la notion de beauté tremblante, délestée de ses atours universalistes, et vectrice fondamentale de changements micro-politiques au niveau mental, social et environnemental. Ce sont ces aspects que les sections suivantes proposent 
d'analyser et de déployer, au travers de ce double paradigme esthétique, en effectuant des détours par la poésie glissantienne.

Du chaos à l'œuvre: vers une poétique du chaos

'L'ordre des mots distrait le monde', ${ }^{33}$ nous dit Glissant dans un vers de son ultime recueil de poésie, Les Grands Chaos, publié en 1993, en plein nouveau désordre mondial (Glissant dirait l'exercice tyrannique d'un système, et non désordre) où les conflits identitaires enflammaient le Tout-monde, jusqu'à son point d'incandescence extrême que nous connaissons actuellement. Aussi beau soit-il, car la première moitié du vers qualifie aussi le bel ordonnancement du poème dont il est issu, 'l'ordre des mots' relèverait d'une pensée de système et d'un système de pensée: langue et idéologie, formes d'écriture et formes d'arts, mesures métriques, syntaxiques et esthétiques etc. Paradoxe aussi que d'émettre du dedans d'un système poétique un souhait d'ouverture vers un dehors absolu. Inextricable proposition qui avère une chose et son contraire: n'est-il pas parfois désirable de distraire le monde? Mais n'est-il pas aussi éthiquement condamnable de dévier notre attention des souffrances réelles du monde? Quel rapport entre la sphère protégée des mots et l'indémêlable chaos destructeur qui agite le monde, l'environnement et des humains qu'on n'ose plus appeler désormais des habitants, tant les frontières et les nationalismes font de victimes? Non, chez Glissant, il arrive souvent que la parole émise ne prime pas le monde, mais en constitue une méprise, une distraction, une arrogance, voire une mutilation (c'est ainsi qu'il parle parfois 'd'agents d'éclat', de tautologies meurtrières retransmises par les médias). C'est dans ce sens négatif et mutilant que le verbe distraire revient souvent sous la plume de Glissant, dans ses formules les plus fondamentales: 'La poétique de la Relation me conduit à la définir

33. Édouard Glissant, 'L'Eau du volcan', Les Grands Chaos, in Poèmes, pp. 453-71 (p. 465). 
comme la quantité finie des différences du monde sans qu'on puisse en distraire la plus infime. 34

Et pourtant, l'œuvre glissantienne repose sur une 'nécessité d'expression', ${ }^{35}$ celle d'un lieu et d'une communauté d'abord, la Martinique, puis les Caraïbes ou l'Antillanité, aux prises avec cet 'impossible à exprimer': le gouffre de la Traite. Rappelons la formule glissantienne: 'structurer le cri [...] crier la structure'. ${ }^{36}$ La distraction serait alors à interpréter comme une stratégie de détour, de désordre: le sens vieilli de 'distraire' ('tirer en divers sens, séparer du tout, dérober' d'après le $C N R T L$ ) nous ramène non à la volonté d'oubli mais aux pratiques de détournement des esclaves marrons, à leur fréquentation de la trace qui les protégeaient de la traque. Distraire le monde, c'est le détourner à son profit par le truchement de cette langue 'parlée-écrite et chantée orale' à laquelle a toujours aspiré Glissant, ${ }^{37}$ c'est faire relation avec la terre, c'est faire poème, qui là, enfin révélé à lui-même en sa diction non impérieuse et non systématique, serait bien un ordre des mots qui distrait le monde tel qu'il est donné et (mal) vécu. L'ordre des mots tout à coup, dans un chiasme glissantien, prime le monde à son tour, et ce n'est pas le système qui est arrogant, aveugle et sourd, mais la fausse évidence du monde. L'ordre chaotique et imprévisible du poème vient bouleverser l'ordre prédictible et déterminé du monde.

La liberté irrépressible du dire fonde en rhétorique - puissance langagière que Glissant ne rejette pas mais change de l'intérieur, loin des résonances prescriptives de la langue aux prétentions universelles et loin des 'rimes coloniales' 38 — la liberté de vivre le lieu. N'oublions

34. Édouard Glissant, 'Le Risque total est de l'enfermement et de la fixité', in Esthétiques du pire, éd. par Richard Conte (Paris: Lienart, 2011), pp. 14-30 (p. 19). C'est nous qui soulignons.

35. Glissant, Discours, p. 343.

36. Philippe Artières, 'Solitaire et solidaire: entretien avec Édouard Glissant', in Pour une littérature-monde, éd. par Michel le Bris et Jean Rouaud (Paris: Gallimard, 2007), pp. 7786 (p. 83).

37. Édouard Glissant, Une nouvelle région du monde (Paris: Gallimard, 2006), p. 111. Voir aussi l'ironique aphorisme: 'La Relation n'affirme pas l'être, sinon pour distraire' ( $P R$ 200).

38. Glissant, 'L'Eau du volcan', Les Grands Chaos, in Poèmes, p. 457. 
pas aussi que c'est par l'expression poétique que Césaire et ensuite Glissant vont se nommer eux-mêmes, c'est-à-dire le je du nous et le nous du je, tout en disant le monde: Le Sang rivé pour l'un, dès 1947, Le Cahier d'un retour au pays natal pour l'autre, dès 1939, tels ces vers où leurs deux voix se confondent: 'Ainsi je fus, colonie d'enfants martyrs de chiens trouvés de squales non convertis. O la souffrance, ce battement de vent dans les rues. La pauvreté est ignorance de la terre, l'imaginé est passion. ${ }^{39}$ L'épigraphe du Sang rivé, 'À toute géographie torturée', est à elle seule un programme éco-poétique qui démantèle les systèmes de la poésie, en inaugurant une forme d'écriture qui travaille à fronts renversés:

\section{À toute géographie torturée}

Non pas l'euvre tendue, sourde, monotone autant que la mer qu'on sculpte sans fin - mais des éclats, accordés à l'effervescence de la terre - et qui ouvrent au cour, pardessus le souci et les affres, une stridence de plages - toujours démis, toujours repris, et hors d'achèvement - non des œuvres mais la matière elle-même dans quoi l'ouvrage chemine tous, liés à quelque projet qui bientôt les rejeta — premiers cris, rumeurs naïves, formes lassées - témoins, incommodes pourtant, de ce projet - qui, de se rencontrer imparfaits se trouvent solidaires parfaitement - et peuvent ici convaincre de s'arrêter à l'incertain - cela qui tremble, vacille et sans cesse devient - comme une terre qu'on ravage - épars ${ }^{40}$

Nul poète ici, nulle œuvre régie par la volonté d'un seul, nul projet achevable projeté sur le monde, mais une série d'éclats qui cheminent dans l'incertain des terres et des peuples ravagés par l'histoire - c'est à ce moment-là que la Martinique devient un département français et que l'oubli des traces de domination est entériné au nom d'une pseudoémancipation économique qui conduira à la destruction de maintes

39. Glissant, Le Sang rivé, in Poèmes, p. 28

40. Glissant, Le Sang rivé, in Poèmes, p. 9. C'est nous qui soulignons. 
ressources naturelles et une hyper ou infra-dépendance vis-à-vis de la 'Métropole'. La terre ravagée, les mémoires des peuples dominés priment désormais la traditionnelle tentative de projet poétique: c'est là ce que Glissant appelle une intention poétique, et qui n'a rien à voir avec une volonté individuelle ou un thème d'inspiration. L'auteur anonyme n'est ni fonction auctoriale justiciable d'un savoir ou d'un pouvoir (l'auctor étant celui 'qui se porte garant de', qui exerce autorité et influence), ${ }^{41}$ ni pure origine: les mots sont des traces inscrites à même un paysage qui n'est plus décor ou (pré)texte, mais une instance d'agencement où viennent affleurer les traces des mémoires africaines disséminées et enfouies et les traces des marrons servant à marquer les chemins de fuite. La signification ne relève pas d'un système linguistique fondé sur la différentiation, mais d'un impossible à exprimer qui s'incorpore pourtant dans ce que Chamoiseau appelle une 'architecture gestuelle liée à des rituels, des danses sacrées que le maître ignorait', ${ }^{42}$ et dans la parole des contes autour de laquelle 'une communauté brisée va s'articuler'. Glissant décrit un processus d'écriture poétique qui ne s'origine ni dans une essence, ni dans une complaisante subjectivité. Les éclats et l'épars, la terre des îles sont la matière chaotique $\mathrm{du}$ poème qui doit s'accorder à eux. ${ }^{43}$ L'éparpillement, la dissémination du sens ne vient donc pas d'une grammatologie, mais d'une (gramma-)thanatologie où ce n'est pas l'auteur qui est mort, mais ce dont il ou elle parle. Le 'sel noir', le 'sang rivé': notons l'amphibologie de chacune de ces formules qui ne sont pas traditionnellement métaphoriques, mais cris et traces des esclavagés. ${ }^{44}$ Le sang 'rivé', c'est-à-dire enchaîné, donnera naissance

41. Voir Paul Audi, L'Autorité de la pensée: essai sur la notion d'auteur (Paris: PUF, 1997).

42. Valérie Marin La Meslée, 'Retrouver la mémoire des corps', entretien avec Patrick Chamoiseau, in La Pensée noire: les textes fondamentaux, Le Point, hors série, avril-mai 2009, pp. 40-41 (p. 40).

43. Dans son Aimé Césaire, frère volcan (Paris: Seuil, 2013), Daniel Maximin parle du poème qui 'est toujours issu d'une obscure émergence, d'une irruption de mots. C'est le poème qui fait le poète et non l'inverse; et le poème confie au poète une faveur d'essentiel cachée au fond des mots sans remous de surface' (p. 50).

44. Hanétha Vété-Congolo, L'Interoralité caribéenne: le mot conté de l'identité. Vers un traité d'esthétique caribéenne (Berlin: Éditions Universitaires Européennes, 2011). 
aux dérives et dévirades glissantiennes par la suite. Ce sont avant tout les marques évanescentes d'une culture archipélique et d'un paysage meurtri. Seul élément d'espoir dans ce thrène: la solidarité des tracestémoins, véritables ritournelles et lignes de fuites guattariennes, c'està-dire des connecteurs et vecteurs de transversalités entre différents milieux (rythme dé- et re-territorialisant), qui sont de fait les premiers germes de la Relation: le poème, c'est déjà la Relation à l'œuvre dans une forme d'écriture où allocuteur et allocutaire se fondent et se confondent en échos.

En filigrane de cette terre gaste se profile une multiplicité de nouvelles formes de subjectivités, les géographies ravagées des Caraïbes devenant le laboratoire réel d'une polyvocalité, de subjectivités plurielles et polyphoniques qui font éclater les systèmes de pensée imperméables à l'altérité et à une conception transversale des identités. Glissant, comme par la suite Guattari dans un tout autre contexte et dont je vais reprendre les termes et les outils conceptuels ici, pressent l'avènement tremblant d'un nouveau paradigme esthétique dans les paysages et les cultures fractals des Caraïbes, infiniment reliés entre eux par cette maintenant fameuse identité-rhizome. Véritable 'agencement collectif d'énonciation' ( $\mathrm{CH} 21)$, le poème fonde le territoire existentiel partiel qui ira, en s'étendant transversalement, répondre à d'autres territoires existentiels partiels dans le Chaosmonde.

Le Chaos-monde et le poème convient tous deux à de nouvelles manières de les vivre et de les naviguer sans chercher à y imposer un ordre ou une mesure rassurants. Pour ce faire, il faut nécessairement renoncer aux pensées de système qui ont 'imposé, ici et là', nous dit encore Glissant, 'un absolu de l'être, qui fut profondeur, magnificence et limitation', ${ }^{45}$ alors même que Guattari met à bas tous les systèmes habituels de pensée (économiques et idéalistes) qui bloquent la libération des subjectivités:

45. Édouard Glissant, Traité du tout-monde (Paris: Gallimard, 1997), p. 20 
Des dualismes en impasse, comme les oppositions entre sensible et intelligible, la pensée et l'étendue, le réel et l'imaginaire, induiront le recours à des instances transcendantes omnipotentes et homogénétiques [...]. La valeur transcendante se pose comme immobile, toujours déjà là et devant toujours le rester. À son égard, la subjectivité reste en manque perpétuel [...] [L]e mensonge de l'idéal devient la malédiction suspendue au-dessus de la réalité. ( $\mathrm{CH}$ 144)

Guattari et Glissant se rejoignent dans leurs conceptions plurielles visant à produire de nouvelles subjectivités effectivement transformatrices et créatives, ce qui implique cependant de composer avec les systèmes existants, une fois ceux-ci rendus erratiques et chaotiques. Si Glissant renonce aux systèmes de pensée, il se refuse aussi à en exclure totalement les fonctionnements et les principes. Chez lui, c'est le fourmillement de toutes les pensées de système qui empêche d'en favoriser aucune, et ce, même s'il ne cesse de les renverser de leur piédestal et d'attaquer leurs fondements. C'est l'aspect le plus complexe de sa pensée, parfois critiqué comme une forme d'accommodement avec les systèmes de domination, alors que c'est l'inverse qui se passe, Glissant étant parfaitement au fait de l'impact colonial, du 'colonialisme qui a pris une autre forme' et qui est intimement lié à celui du Capital $^{46}$ — les systèmes de pensée continentale — sur les situations d'aujourd'hui. Si d'un côté toute critique émanant de l'intérieur d'un système se voit obligatoirement conduite à reproduire un tant soit peu ce système, prétendre à une pensée pure de tout système relèverait d'une arrogance similaire. À plusieurs reprises, Glissant n'hésitera pas à brouiller les cartes de ses propres déclarations, les modifiant, les revisitant de telle sorte qu'il les sort d'une facile sédimentation dans un sens univoque ou trop systématique justement ('Ce n'est pas le système qui est récusable. Ce qui est récusable, c'est que le système soit systématique'), ${ }^{47}$ revenant sur la nécessité de créer son système pour faire opposition aux autres,

46. Glissant, L'Imaginaire, p. 65.

47. Glissant, L'Imaginaire, p. 77. 
mais sans le laisser à son tour sombrer dans une dérive hégémonique et fermée au chaos du vivant.

Suivant une même stratégie d'écriture, Glissant reviendra dans une lettre à Alexandre Leupin sur la nécessité du concept, d'obédience hégélienne, mais libéré de son enfermement systématique par la puissance de l'imaginaire (sans doute d'origine sartrienne et à ne pas confondre avec l'imagination kantienne): 'Sur l'imaginaire. Ce par quoi le concept échappe à son enfermement, c'est-à-dire à son propre système. ${ }^{48} \mathrm{D}$ 'où le besoin de ferrailler avec ces systèmes, mais sans hésiter à en prendre la mesure lorsque la situation l'exige: 'le Toutmonde est une démesure et si nous ne prenons pas la mesure de cette démesure nous risquons, c'est une des bases de ma poétique, nous risquons de trainer et traîner les vieux impossibles qui déterminent toujours les mêmes intolérances, massacres, génocides' ( $P A$ 152). Le terrain sur lequel la pensée glissantienne se fonde est de toute façon sismique et imprévisible, inutile donc de la 're-continentaliser'. Protégée d'un voile d'opacité, la pensée glissantienne ne se laisse vraiment approcher que lorsqu'elle s'étend concrètement en poésie, et se traduit en poétiques. De son côté, Guattari compose ('composer' étant pris ici dans le sens musical du terme) aussi avec les 'vieux' systèmes - pas question d'esthétiser la vie, mais nécessité de faire émerger du socius des formes d'altérité, des potentialités actualisables en situation - en créant de nouveaux systèmes de valorisation (singularisation, devenirs intensifs et processuels) comme vecteurs d'hétérogenèse:

Le pli chaosmique de déterritorialisation et le pli autopoïtique d'énonciation [...] implantent au cœur du rapport objet-sujet, et en deçà de toute instance de représentation, une processualité créative, une responsabilité ontologique qui noue la liberté et son vertige éthique au cœur des nécessités éco-systémiques. (CH 174)

48. Lettre reproduite en exergue de Leupin, Édouard Glissant. 
Guattari n'hésite pas non plus à mettre la production poétique en avant, comme étant seule capable de rompre avec les systèmes de connaissance dominants. La poésie est ce qui permet d'appréhender (non de comprendre ou maîtriser), c'est-à-dire de deviner et de mettre en œuvre les nouvelles subjectivités respectueuses du monde et de l'altérité: 'la poésie, aujourd'hui, a peut-être plus à nous enseigner que les sciences économiques, les sciences humaines et la psychanalyse réunies!' (CH 39).

\section{Le poème de la beauté tremblante}

C'est dans cette perspective qu'il faut comprendre pourquoi, chez Glissant, le poème est toujours premier ('cela commence toujours par un poème' est une ritournelle glissantienne), même si ce qu'il entend par poème sort des catégorisations existantes. Comme il nous le dit dans Philosophie de la relation, sous-titré Poésie en étendue, le poème est l'un des invariants du chaos-monde, sans pour autant offrir de certitude: 'Le poème est la seule dimension de vérité ou de permanence ou de déviance qui relie les présences du monde. ${ }^{49}$ Cette pensée du poème ne cesse de s'incarner dans ses textes, de façon soit répétitive (induisant d'invisibles changements) soit surprenante (formant synthèse, ouverture, accompagnement etc. par rapport au texte). C'est le cas d'un 'proême' qui se trouve lové sous plusieurs moutures dans La Cohée du Lamentin, et qui étend tout à la fois un appareil conceptuel et une rêverie méditative, qui ne répond pas d'une subjectivité ou d'une autorité particulière, mais à un déploiement de modes de subjectivation:

La pensée du tremblement éclate partout, avec les musiques et les formes suggérées par les peuples. Elle nous préserve des pensées de système et des systèmes de pensée. Elle ne suppose

49. Édouard Glissant, Philosophie de la relation: poésie en étendue (Paris: Gallimard, 2009), pp. 19-20. 
pas la peur ou l'irrésolu, elle s'étend infiniment comme un oiseau innumérable, les ailes semées $d u$ sel noir de la terre. Elle nous unit dans l'absolue diversité, en un tourbillon de rencontres. Elle est l'utopie qui jamais ne se fixe et qui ouvre demain: comme un soleil ou un fruit partagés. ${ }^{50}$

Ce texte se place d'entrée de jeu sous l'égide d'un agencement collectif d'énonciation incarné par le pronom 'nous' qui obsède jusqu'à l'accablement Glissant depuis ses premiers poèmes du Sel noir justement, sel du deuil et de la plaie souffrante, sel des plages du Lamentin ou de Carthage détruite, mais aussi sel fertile d'au-dessous $\mathrm{du}$ volcan, que l'oiseau, indéfiniment varié et irrémédiablement marqué, sème à tout vent. La pensée du tremblement est le signe de sa fragilité anti-systémique comme des bouleversements imprévisibles qu'elle induit dans le champ du possible; elle est tout aussi bien esthétique ('musiques et formes des peuples'), éthique ('soleil et fruits partagés') et politique ('unit dans l'absolue diversité') sans pour cela s'embarrasser de normes prescriptives et de fixités idéologiques ('utopie qui jamais ne se fixe'). Le tremblement relève du courage de la poésie qui ne perd pas la trace de la vulnérabilité des dominés, mais il procède aussi du chaos écologique du monde. Le tremblement est la force créatrice et destructrice qui révèle les structures invisibles et invite à les ausculter. La pensée du tremblement relie sujet et objet sans qu'ils se confondent: c'est un mode de connaissance opératoire, une poétique concrète qui rejette la mimésis du réel au profit d'une écriture de la trace (qui marronne, se dérobe et dérobe au réel fixe): 'l'ordre des mots' peut ainsi servir à ausculter les tremblements du monde, à les fixer dans une forme qui n'en occulte pas pour autant l'énergie chaotique. Glissant reprendra cette idée dans un article peu cité et publié dans un livre d'éco-esthétique au titre évocateur Esthétiques $d u$ pire:

Quand l'écriture tremble, elle raffermit sa matière et parfois même lui découvre des structures inattendues [...]. À chaque

50. Glissant, La Cohée du Lamentin, p. 33. C'est nous qui soulignons. 
moment l'artiste risque d'effacer le tremblement sous la fixité, mais que, lorsqu'il échappe à ce risque, la fixité formelle est d'autant plus belle que le tremblement par-dessous anime la vie d'une matière, qui est celle du monde, que nous courons à notre tour le risque de la toucher [...]. Le tremblement de la pensée de l'art est l'œuvre de magie par laquelle ces humanités courent le risque de savoir aller au-delà de cette indifférence de la terre pour nous. ${ }^{51}$

Le vrai risque, la seule abdication possible, du côté du créateur comme de 'l'artmateur', serait de ne pas courir jusqu'au bout le risque de l'œuvre, d'en effacer les différences chaotiques pour sombrer dans l'indifférenciation, un spectacle du beau qui nous serait et laisserait finalement, et à juste titre, indifférents. La beauté, concept-notion prégnant chez Glissant, échappe aux tentatives de la circonscrire dans un système d'esthétique, et sert au contraire d'horizon d'espoir, horizon sans cesse créé et détruit. La beauté relève d'une connaissance à vif, toujours collective et partagée, et n'appartient à aucun domaine privilégié. La beauté fait gripper les systèmes bien huilés et elle se joue d'eux. Elle a partie liée avec des 'liaisons magnétiques', 52 des formes de communion qui n'ont pas trouvé leur place dans les esthétiques sédimentées connues, excepté parfois celles des (pré-)romantiques allemands (Hölderlin et Schiller surtout) et d'Artaud. C'est ce qui fait dire à Glissant que

Le tremblement sous la fixité ouvre sur la naissance de la beauté $[\ldots]$

[L]e tremblement est l'énergie de l'art, parce qu'il est l'énergie du monde $[\ldots]$.

Le risque majeur de l'art, le risque total, est l'art lui-même [...]. La pensée du tremblement court le risque de s'opposer aux pensées de système, et aux systèmes de pensée, s'accordant

51. Glissant, 'Le Risque total est de l'enfermement et de la fixité', p. 17

52. Glissant, Région, p. 48. 
peut-être à cette proposition que je formule ici 'rien n'est vrai; tout est vivant ${ }^{53}$

Qu'est-ce à dire? Glissant n'introduit-il pas là un système plus subtil sous couvert d'un renoncement apparent aux systèmes de pensée qui ont défini l'Occident? À l'instar de Guattari, son tourbillon de concepts-notions nous convie à les questionner et à en questionner les tremblements sous-jacents: la pensée se fait transversale, son expression est relationnelle et hors du système 'dictatorial' du signifiant (elle est a-signifiante) ${ }^{54}$ et de la subjectivité consacrée; elle procède par contamination, prolifération rhizomique, débranchements et branchements multiples entre l'humain et le non-humain. Ces deux penseurs travaillent, et sont travaillés par, la matière même du chaosmonde, au moment où la chaosmose atteint son point critique, aux alentours des années 1990. Résolument interdisciplinaires et indisciplinés, ils n'hésitent pas à mettre en avant la fonction poétique pour l'un, l'intention poétique pour l'autre, seules capables selon eux de faire opposition aux nouvelles formes d'hégémonie impériale. À un moment où l'esthétique était devenue synonyme de complaisance et de désengagement politique, Guattari en appelle à l'instauration d'un 'nouveau paradigme esthétique' qui, pour lui, a des implications éthico-politiques 'parce que parler de création, c'est parler de responsabilité de l'instance créatrice à l'égard de la chose créée [...] bifurcation au-delà des schémas préétablis, prise en compte [...] du sort de l'altérité dans ses modalités extrêmes. Mais ce choix éthique n'émane plus d'une énonciation transcendante' ( $\mathrm{CH}$ 149-50). Selon Pascale Criton, 'Il ne s'agit pas d'esthétiser la vie, ni de faire entrer

53. Glissant, Esthétiques du pire, p. 17.

54. Voir l'analyse de Stephen Zepke, sur l'a-signifiance du signe de l'art tel qu'il est envisagé par Guattari: 'Such signs — produced by art — create sensations that escape their subsumption by individualized, subjective experience and signification to express a "collective assemblage of enunciation" encompassing all its constitutive elements, human and nonhuman.' Stephen Zepke, 'From Aesthetic Autonomy to Autonomist Aesthetics: Art and Life in Guattari', in The Guattari Effect, éd. par Éric Alliez et Andrew Goffey (London: Continuum, 2011), pp. 205-19 (p. 207). 
l'art par les pores du quotidien [...] la fonction éthico-poétique est l'aiguillon d'une autre conception de la vie [...] qui n'écrase pas la différence. ${ }^{55}$ Dans une belle conjointure de pensée, ${ }^{56}$ Glissant et Guattari s'accordent sur la nécessité de concevoir à nouveaux frais les modes de production de la subjectivation, foncièrement hétérogénétiques, selon des compositions autopoïétiques (terme que Guattari emprunte à Varela), 'qui engendrent et spécifient continuellement leur propre organisation', ce qui implique de faire justice des divisions disciplinaires et conceptuelles courantes, quand bien même celles-ci seraient remplies de bonnes intentions.

La pensée du tremblement ouvre à des esthétiques plurielles qui nous changent, à défaut de changer directement les choses, stade liminaire où l'utopie glissantienne et guattarienne commence, puisqu'elle n'est que supposition et n'infère aucun résultat prédictible. L'esthétique n'en sera pas bannie, ni les poétiques qui en sont les applications concrètes à travers les arts, ni la beauté qui les inclut tout en les débordant absolument, dans les géographies et paysages notamment, renversant au passage les systèmes occidentaux du beau, en commençant par Hegel, et sans oublier la façon dont Glissant, à l'instar de Bonnefoy, choisit contre Platon la plénitude du monde et ne s'effraie pas de ses ombres. Si d'un côté Guattari 'renouvelle [profondément] le sens du terme esthétique qui [désormais] ne définit plus une catégorie étanche et localisée dans la sphère du beau', ${ }^{57}$ Glissant nous avertit de ne pas

55. Pascale Criton, 'L'Esthétique déterritorialisée', Chimères, Numéro spécial, 'Chaosmose, penser avec Félix Guattari', 77 (2012), 25-34 (p. 32).

56. On pourrait objecter une différence irréductible entre le créateur de la schizoanalyse, dont les recherches portent sur la dé-subjectivation, et Glissant, dont la réserve envers la psychanalyse et sa défense du sujet sont connues, mais ce serait confondre une différence de mots et une affinité de préoccupations: les deux penseurs évident le sujet du 'désert du réel' qui parade en parc d'attraction de la subjectivité festive, au profit de singularisations et d'individuations basées sur des prémisses similaires. Fanon reste de toute façon un lien intellectuel vital entre ces deux penseurs anti-colonialistes.

57. Criton, 'L'Esthétique déterritorialisée', p. 32. 
[confondre médiocrement] la beauté, lieu-commun des rencontres des différences, et le spectacle du beau, qui en a toujours été le figement [...]. Le beau en chaos y suggère les possessions inspirées de la beauté, mais il le fait si mécaniquement [...]. Ses cadences en effet ne répètent pas, c'est redondance neutre: (il suffit qu'elles nous distraient et nous engourdissent). ${ }^{58}$

Nous voilà retourné à ce 'distraire' du poème tiré de Les Grands Chaos: 'l'ordre des mots', aussi beau soit-il, dans une syntaxe/métrique ou signification des plus subtiles, ne fait effectivement que distraire le monde et le transformer en spectacle divertissant du beau. Seule la matière qui sourd en frémissant dans la forme, dans l'impeccable fixité du beau, ouvrirait à la naissance de la beauté. Mais ce vers, ' 1 'ordre des mots distrait le monde', cité comme parole sibylline de 'L'Eau du volcan', était en fait une reprise d'un vers issu d'une section précédente du même recueil, et celui-ci disait tout son contraire: 'l'ordre des mots ne distrait pas le monde',59 c'était alors dans un contexte de parole tout autre, lieu mythologique et rêvé où 'poètes et conteurs y font exprès d'oblitérer la page ou de taire l'acclamation': ce monde-là des liaisons secrètes (dieux du Nil et des îles) ne peut être perturbé par aucun langage, c'est le chaos de la pure 'tremblance'. Ce contraire tout grammatical en surface correspond à une concordance de fond: 1'ordre des mots n'a aucune prise sur le monde (ce qui ne veut pas dire que l'ordre du poème, toujours caché, ne puisse rien en dire). En cette fin du dernier livre de poésie écrit par Glissant, l'eau du volcan, les sables noirs des îles, n'admettent plus l'ordre des mots, et l'injonction, qu'elle soit à l'affirmative ou à la négative, démet toute prétention ordonnatrice des mots et de leur système: $d$ 'autres langages sont encore à naître, totalement démesurés. C'est du moins ce que suggère le poète driveur, qui recueille le dit de l'ombre, parole première de cette catabase, forme glissantienne d'un retour au pays natal révélé dans ce qui lui est propre:

58. Glissant, Philosophie, pp. 76-77. C'est nous qui soulignons.

59. Glissant, 'L'Eil dérobé', Les Grands Chaos, in Poèmes, p. 433. 
Il fouilla Morne et sables morts. Et sous le sable, morts Vaquaient les mots, tortus d'être vacants.

[...]

L'écho descendant, ah! déhalait son double, qui ainsi criait.

'Il est des mots qui brûlent en leur lieu, ils ne servent qu'à une fois.

Ils ne paraissent à métier ni à beauté d'usage,

Et n'est de langue pour pays, où tous langages s'émerveillent

L'ordre des mots distrait le monde...'

Il descendit dans la houlée du bois. ${ }^{60}$

Le poème permet, dans le retrait qu'il fait du monde, cette formulation paraliptique, impossible et pourtant nécessaire: dire le monde sans le dire. En nul autre nom, que celui des souffles inaudibles et enfouis qui hantent nos mots et nos langages, mais aussi que celui que tous nos langages sauront célébrer. C'était là peut-être ce que voulait dire Walter Benjamin, ce migrant vulnérable qui est mort lui aussi d'une frontière par trop étanche, lorsqu'il replongeait les mots et leurs systèmes dans le silence qui borde tout poème: 'L'inexpressif force l'harmonie frémissante à suspendre son vol et, par cette protestation, en éternise le tremblement. ${ }^{61}$

\section{Magdalene College, Cambridge}

60. Glissant, 'L'Eau du volcan', Les Grands Chaos, in Poèmes, p. 465.

61. Walter Benjamin, 'Les Affinités électives de Goethe', in Euvres I (Paris: Gallimard, 2000), trad. de Maurice de Gandillac, Rainer Rochlitz et Pierre Rusch, pp. 274-395 (p. 363). Je voudrais remercier de tout cœur Marion Schmid, Jean-Luc Tamby, JeanPascal Pouzet, Celia Britton, Maeve McCusker, Maria Scott, Manuel Norvat et Irving Goh. 